\title{
INTERPOLACIÓN DE VARIABLES DE FERTILIDAD DE SUELO MEDIANTE EL ANÁLISIS KRIGING Y SU VALIDACIÓN ${ }^{1}$
}

\author{
Carlos Henríquez $z^{2 * * *}$, Juan Carlos Méndez*, Ramón Masís *
}

Palabras clave: Geoestadística, SIG en agricultura, cooperativas agrícolas, fertilidad de suelos, validación cruzada, validación de campo.

Keywords: Geostatistics, GIS in agriculture, agri-Cooperatives, soil fertility, cross validation, field validation.

Recibido: 21/01/13

RESUMEN

Con el fin de elaborar y validar los mapas de interpolación de 6 variables de fertilidad, se recolectaron y analizaron 138 muestras de suelo de 1011 ha en la zona de Atirro, Costa Rica. Los resultados fueron interpolados mediante el método geoestadístico Kriging ordinario para las variables $\mathrm{Ca}, \mathrm{pH}$, acidez, $\mathrm{K}, \mathrm{P}$ y porcentaje de saturación de acidez. La validación de los mapas interpolados se llevó a cabo mediante las técnicas "validación de campo" y "validación cruzada". Para ambas técnicas se estimó el coeficiente de correlación (r) entre los valores reales y los de predicción, así como la eficiencia de predicción (E). Adicionalmente se utilizaron 2 criterios para la validación, que fueron el porcentaje de acierto por traslape entre los valores reales y los estimados, según la incertidumbre del análisis de suelos y según un ámbito agronómico. Los coeficientes de correlación obtenidos entre los valores reales y los de predicción mediante la técnica de validación de campo variaron de 0,09 a 0,87 , y para validación cruzada entre 0,52 y 0,84 . Las variables $\mathrm{Ca}$ y $\mathrm{pH}$ fueron las que presentaron las mayores eficiencias de predicción para ambos métodos de validación. El criterio de traslape por

1 Resultados parciales del proyecto VI-733-A7-609 de la Universidad de Costa Rica. Proyecto cofinanciado por el Instituto de Fomento cooperativo (INFOCOOP).

2 Autor para correspondencia. Correo electrónico: carlos.henriquez@ucr.ac.cr
Aceptado: 03/07/13

\section{ABSTRACT}

Interpolation of soil fertility data with Kriging and its validation. The goal of this study was to make and validate interpolated maps of 6 soil fertility variables. The maps were made from the results of $\mathrm{Ca}, \mathrm{pH}$, soil acidity, $\mathrm{K}, \mathrm{P}$ and saturation of soil acidity of 138 soil samples that were taken from 1011 ha at Atirro, Costa Rica. The data were interpolated through ordinary Kriging. The validation was carried out using "field validation" and "cross validation" methods. Correlation coefficient (r) was estimated for both techniques between real and prediction values, and the prediction efficiency (E) as well. Other validation criteria used were the percent success by overlapping between real and estimated values, according to the uncertainty of soil analysis and to the success rate of overlap according to agronomic category. The $\mathrm{r}$ values using field validation varied from 0.09 to 0.87 ; and for cross validation were from 0.52 to 0.84 . The variables $\mathrm{Ca}$ and $\mathrm{pH}$ had the highest prediction efficiency in both validation methods. The overlap criterium due to the uncertainty of analysis was 27 to $93 \%$ success, while the overlapping range that was due to agronomic category had 47 to $93 \%$ of success.

\footnotetext{
* Centro de Investigaciones Agronómicas, Universidad de Costa Rica (CIA-UCR).

** Sede del Atlántico, Universidad de Costa Rica (CIA-UCR).
} 
la incertidumbre del análisis fue de 27 a $93 \%$ de acierto, mientras que por clase agronómica los resultados de verificación oscilaron de 47 a 93\%, y en ambos casos la variable $\mathrm{pH}$ fue la que mayor porcentaje de acierto mostró. Al considerar los promedios generales, solo el valor de predicción para la variable $\mathrm{K}$, estuvo levemente por debajo del valor de verificación. Se concluye que los mapas interpolados a escala regional, son una herramienta útil para predecir con un buen grado de acierto las propiedades de fertilidad de suelos; pese a ello, es importante un proceso de verificación para confirmar estas aproximaciones.

\section{INTRODUCCIÓN}

Una de las utilidades más importantes que poseen los Sistemas de Información Geográfica (SIG) es el análisis espacial, en particular el uso de las interpolaciones de diferentes tipos de variables. En el campo agrícola, el uso de esta herramienta permite analizar la variabilidad de diferentes características sobre el paisaje tales como suelo (Clay et ál. 2007), enfermedades y plagas entre otras, lo cual sin duda ayuda a cuantificar el impacto de esta variación sobre la producción y las posibles pautas de manejo requeridas para optimizar los rendimientos (Bertsch et ál. 2002, Henríquez et ál. 2005, Petersen et ál. 1995).

Entre los métodos más utilizados para llevar a cabo la interpolación de variables está la Ponderación del Inverso de la Distancia (IDW por sus siglas en Inglés), método matemático que utiliza un algoritmo basado en las distancias de los puntos. Por otro lado, Kriging es un método geoestadístico que cuantifica la estructura espacial de los datos y su autocorrelación, mediante variogramas para realizar las predicciones correspondientes (Trangmar et ál. 1985, Villatoro et ál. 2008).

En varios estudios el método de interpolación Kriging ha mostrado ser eficiente en la
In both cases, $\mathrm{pH}$ had the better values of success. It was concluded that the interpolated maps at a regional scale are a useful tool for to carrying out a good prediction on soil fertility properties, although it is important to perform a verification process in order to confirm these approximations, because this could change according to the type of variables.

discriminación de áreas con diferentes características de importancia agronómica. En la agricultura de precisión esto es de mucha utilidad para mejorar las prácticas de manejo, acordes con la variabilidad encontrada (Petersen et ál. 1995, Henríquez et ál. 2005).

Villatoro et ál. (2008) encontraron que el método Kriging mostró, según el análisis de efectividad de predicción (E), mejor grado de estimación para variables como el $\mathrm{pH}$, Ca y CICE y en menor grado para $\mathrm{P}$, en comparación con el IDW. La ventaja que tiene el método geoestadístico con respecto al matemático es la verificación de la validez de la interpolación realizada, a través de parámetros estadísticos provistos por el análisis.

El uso de los mapas de interpolación generados a partir de las variables químicas reportadas en un análisis de suelos ha sido catalogado muy útil en el diagnóstico y recomendación de fertilización y aplicaciones de enmiendas en Costa Rica. Ejemplo de ello es lo reportado por Bertsch et ál. (2002), quienes lograron establecer un programa de manejo de la fertilización de acuerdo con las necesidades de cada sitio en fincas sembradas con café y caña de azúcar.

Pese a lo anterior, es importante evaluar y comparar los valores de predicción para cada variable con datos reales en puntos específicos antes de adoptar cualquier medida de manejo 
basada en los mapas de interpolación (Robinson y Metternicht 2006). Este proceso de verificación se puede realizar por medio de un análisis de validación, el cual puede ser llevado a cabo de diversas formas. Según Mueller (2007) y Gallardo (2006), una de las formas consiste en la recolección de una serie de datos diferente a la que se utilizó para hacer la interpolación inicial y de esta forma comparar los valores estimados con los medidos en campo en cada uno de los puntos de validación. Este método corresponde a una validación de campo posterior a la elaboración de los mapas interpolados.

Otro método de verificación, es el conocido como "validación cruzada", que consiste en eliminar un dato de la variable evaluada para así generar un valor de predicción en la localidad del punto removido y así las veces como datos existan. Al realizar esta acción con cada uno de los puntos que conforman el grupo de datos originales, se puede obtener una correlación, en la cual se representan los valores interpolados frente a los valores reales (Tomczak 1998, Gallardo 2006).

Por otro lado, existe evidencia que demuestra que los valores de análisis químicos por sí mismos, tienen un ámbito de variación dentro del laboratorio; éste ámbitodepende de cada elemento o determinación (Corrales et ál. 2005). De esta forma es esperable también, que las estimaciones o validaciones, estén supeditadas a esta otra fuente de variación, motivo por el cual en este trabajo también se incorpora este criterio de validación de las interpolaciones. Por otro lado los niveles críticos de los elementos así como de otras características químicas del suelo ( $\mathrm{pH}$ por ejemplo), han sido definidos de acuerdo con ámbitos que definen su estado como deficiente, óptimo o bien elevado; estos ámbitos se han definido en función de clases con aplicabilidad agronómica, en tanto son condiciones en función del crecimiento de la planta (Bertsch 1995). Estos 2 criterios deberían ser tomados en cuenta también en la designación e interpretación de mapas de variabilidad espacial de estas características.

El presente estudio tuvo como objetivo aplicar un proceso de validación a partir del análisis de interpolación de 6 variables de fertilidad del suelo $(\mathrm{Ca}, \mathrm{pH}$, acidez, $\mathrm{K}, \mathrm{P}$ y porcentaje de saturación de acidez) realizado con el método Kriging ordinario, a través de las técnicas de validación por puntos de control o validación de campo y el análisis de validación cruzada, también se utilizaron 2 criterios adicionales de verificación que fueron el porcentaje de acierto con respecto a los ámbitos de variación del laboratorio y por clases agronómicas, ambos haciendo una comparación entre los valores reales y los estimados, esto en las fincas de 3 cooperativas en la zona de Atirro, Costa Rica.

\section{MATERIALES Y MÉTODOS}

\section{Ubicación y descripción general del área de estudio}

El estudio se desarrolló en la localidad de Atirro, provincia de Cartago, Costa Rica (punto de referencia general $9^{\circ} 49^{\prime} 30^{\prime \prime} \mathrm{N}, 83^{\circ} 39^{\prime} 30^{\prime \prime} \mathrm{O}$ ). Las fincas estudiadas se extienden en 1.011 ha, las cuales pertenecen a las cooperativas AgroAtirro R.L. (255 ha), CoopeCañita R.L. (396 ha) y FECOOPA R.L. (360 ha). Las fincas de las 3 cooperativas colindan entre sí y están dedicadas a la producción del cultivo de caña de azúcar y café (Figura 1).

La zona presenta una topografía variable, con altitudes que van desde 640 hasta $960 \mathrm{msnm}$, con sectores planos cercanos a los ríos Pejibaye, Reventazón y Atirro y otros con pendientes pronunciadas en las laderas de los cerros Atirro y Fila Omega. Con respecto a los tipos de suelo, según el sistema de clasificación de suelos del USDA (2010) estos pertenecen al orden de los Inceptisoles (de origen aluvial en las partes planas) y Ultisoles (en las laderas y áreas escarpadas). 


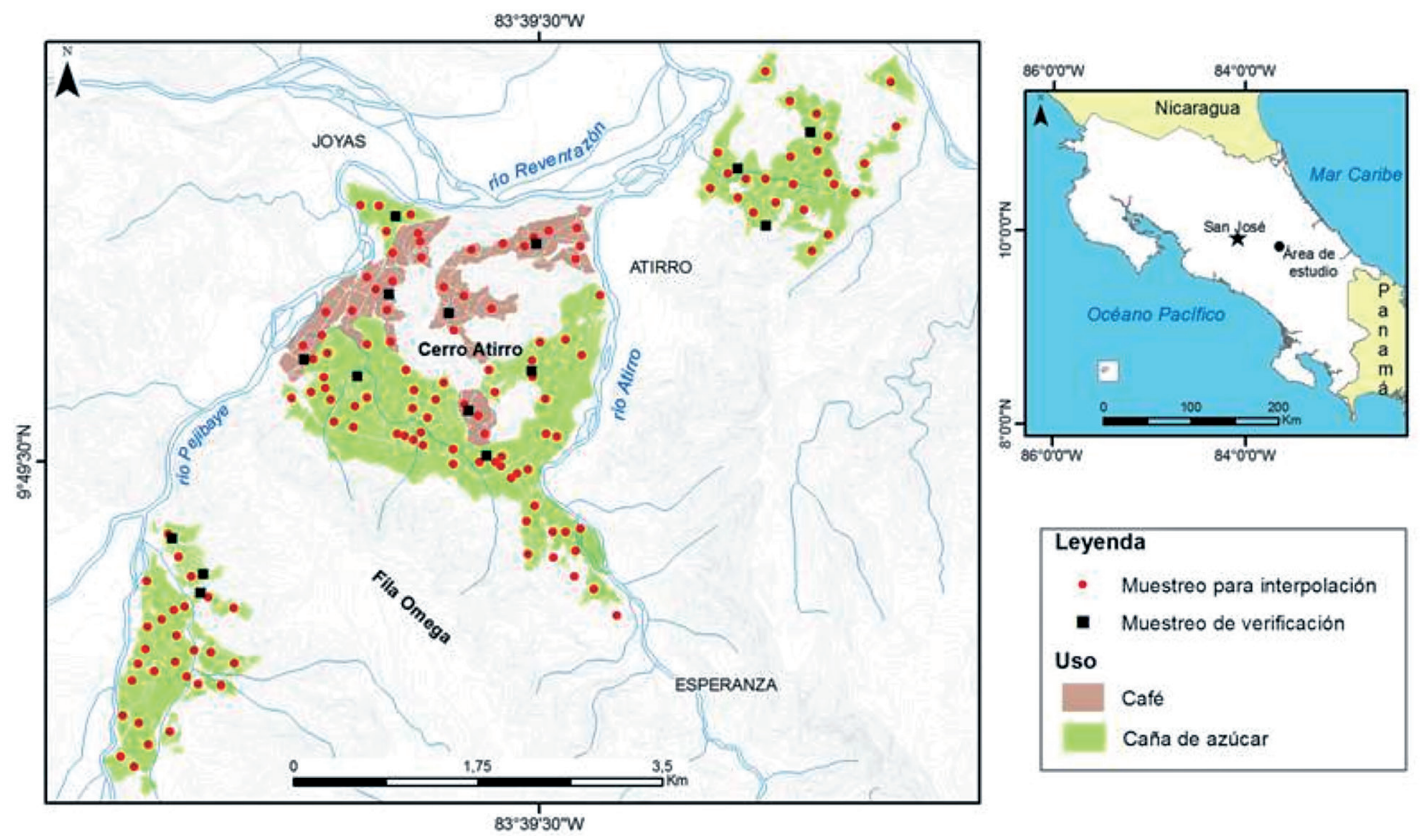

Fig. 1. Ubicación de los puntos de muestreo de suelo usados en la interpolación así como de los puntos de verificación en el área de estudio.

\section{Delimitación de las unidades de muestreo de suelos para el análisis de interpolación}

Los lotes de las fincas fueron delimitados según las unidades de manejo establecidas por cada cooperativa. Para el mapeo se utilizaron fotografías aéreas (CARTA 2005) y mediante un receptor de GPS marca Trimble (Juno SD) se tomaron puntos de referencia y verificación en el campo. Para la georeferenciación de los lotes se utilizó el programa ArcGis (versión 10). Las interpolaciones se hicieron con el programa GS Plus (versión 9). En cada lote se recolectó al menos una muestra de suelo, la cual estuvo constituida por 15 submuestras tomadas a una profundidad de $20 \mathrm{~cm}$ y distribuidas aleatoriamente en el área respectiva de cada lote. En total se recolectaron 138 muestras de suelo, las cuales conformaron el grupo de datos inicial con el cual se elaboraron los mapas interpolados con las variables elegidas. Para ubicar espacialmente cada muestra se asignó un centroide en el polígono correspondiente a cada lote, con el fin de obtener las coordenadas geográficas $(\mathrm{X}, \mathrm{Y})$ y anexar los valores $\mathrm{Z}$ (variables químicas del suelo analizadas), necesarios para realizar las interpolaciones.

\section{Muestreo de suelos para la obtención de los puntos de verificación}

Posteriormente, en forma independiente al muestreo realizado para el análisis de interpolación, se procedió a recolectar un total de 15 muestras (segundo grupo de datos) en puntos distribuidos en forma representativa dentro del área de estudio, con el fin de confrontar los resultados obtenidos del análisis de interpolación mediante la técnica de validación de campo. Para ello se georeferenció en el campo cada punto de muestreo y en un radio no mayor a los $10 \mathrm{~m}$ de cada sitio se recolectaron 15 submuestras a la misma profundidad de muestreo con que se realizó el análisis de interpolación. En ambos muestreos se dieron casos en que la distancia mínima fue de menos de 200 metros (Figura 1). 


\section{Análisis químico de las muestras de suelos}

Las muestras de suelo para llevar a cabo tanto la interpolación como la validación, se llevaron al Laboratorio de Suelos y Foliares del Centro de Investigaciones Agronómicas (CIA) de la Universidad de Costa Rica (UCR). Estas fueron secadas a $60^{\circ} \mathrm{C}$ durante $24 \mathrm{~h}$ y tamizadas por una malla de $2 \mathrm{~mm}$ luego de lo cual fueron analizadas químicamente utilizando las soluciones extractoras Olsen Modificado (K, P) y $\mathrm{KCl} 1 \mathrm{M}$ (Ca, acidez intercambiable) según la metodología descrita por Díaz-Romeu y Hunter (1978). El pH fue obtenido en agua en una proporción de 1:2,5 y el porcentaje de saturación de acidez se calculó a partir de la fórmula (acidez)*(CICE $\left.{ }^{-1}\right)^{*}(100)$.

\section{Análisis estadístico y de verificación}

Los resultados de los análisis químicos correspondientes a las 138 muestras de suelo fueron sometidos al análisis geoestadístico para las variables de estudio. Inicialmente los datos tabulados en coordenadas $\mathrm{X}, \mathrm{Y}$ y Z fueron procesados con el programa GS Plus (versión 9.0) con el fin de obtener los parámetros del semivariograma que mejor ajustaran y correlacionaran espacialmente según cada variable estudiada (Cuadro 1). Posteriormente con los parámetros geoestadísticos obtenidos, se interpolaron los datos por medio del método kringing ordinario a través del programa
ArcGIS (versión 10), con la extensión Geostatistical Analysis, y con el resultado de una superficie continua de la variable interpolada (Figura 2).

Con el fin de estimar la validez de los mapas obtenidos por medio del análisis de interpolación, se realizó la validación de los mismos por medio de 2 técnicas diferentes. En primer lugar y con el uso del segundo grupo de datos recolectado, se aplicó el análisis de "validación de campo", el cual consiste en comparar los valores obtenidos en el muestreo de verificación realizado en diferentes puntos con aquellos que se obtuvieron producto de la interpolación en esos mismos puntos. La otra técnica utilizada fue "validación cruzada", en la cual se utilizó únicamente el grupo de datos inicial (138 datos) que dio origen a las interpolaciones. Este proceso implica la remoción de un dato del grupo original para posteriormente realizar una predicción del valor de la variable en la localización del dato removido y así sucesivamente cuantas veces como datos existan. Este proceso es realizado con todos los datos de los puntos observados con los valores estimados.

Para ambos métodos de validación se determinó el coeficiente de correlación (r) entre los valores obtenidos y los de predicción así como el valor de eficiencia de predicción (E), el cual es una medida de efectividad de la predicción de

Cuadro 1. Parámetros geoestadísticos utilizados para 6 variables de suelo, Atirro, Costa Rica.

\begin{tabular}{|c|c|c|c|c|c|}
\hline Variable & Modelo & Nugget & Sill & Range & $\mathrm{R}^{2}$ \\
\hline $\mathrm{pH}$ & Esférico & 0,11 & 0,31 & 2480 & 0,79 \\
\hline $\mathrm{Ca}$ & Esférico & 9,8 & 55,84 & 2332 & 0,82 \\
\hline $\mathrm{K}$ & Gausiano & 0,002 & 0,017 & 500 & 0,55 \\
\hline $\mathrm{P}$ & Esférico & 235 & 619 & 1351 & 0,74 \\
\hline Acidez & Gausiano & 0,17 & 1,72 & 447 & 0,68 \\
\hline$\% \mathrm{SA}$ & Gausiano & 0,10 & 230 & 415 & 0,55 \\
\hline
\end{tabular}



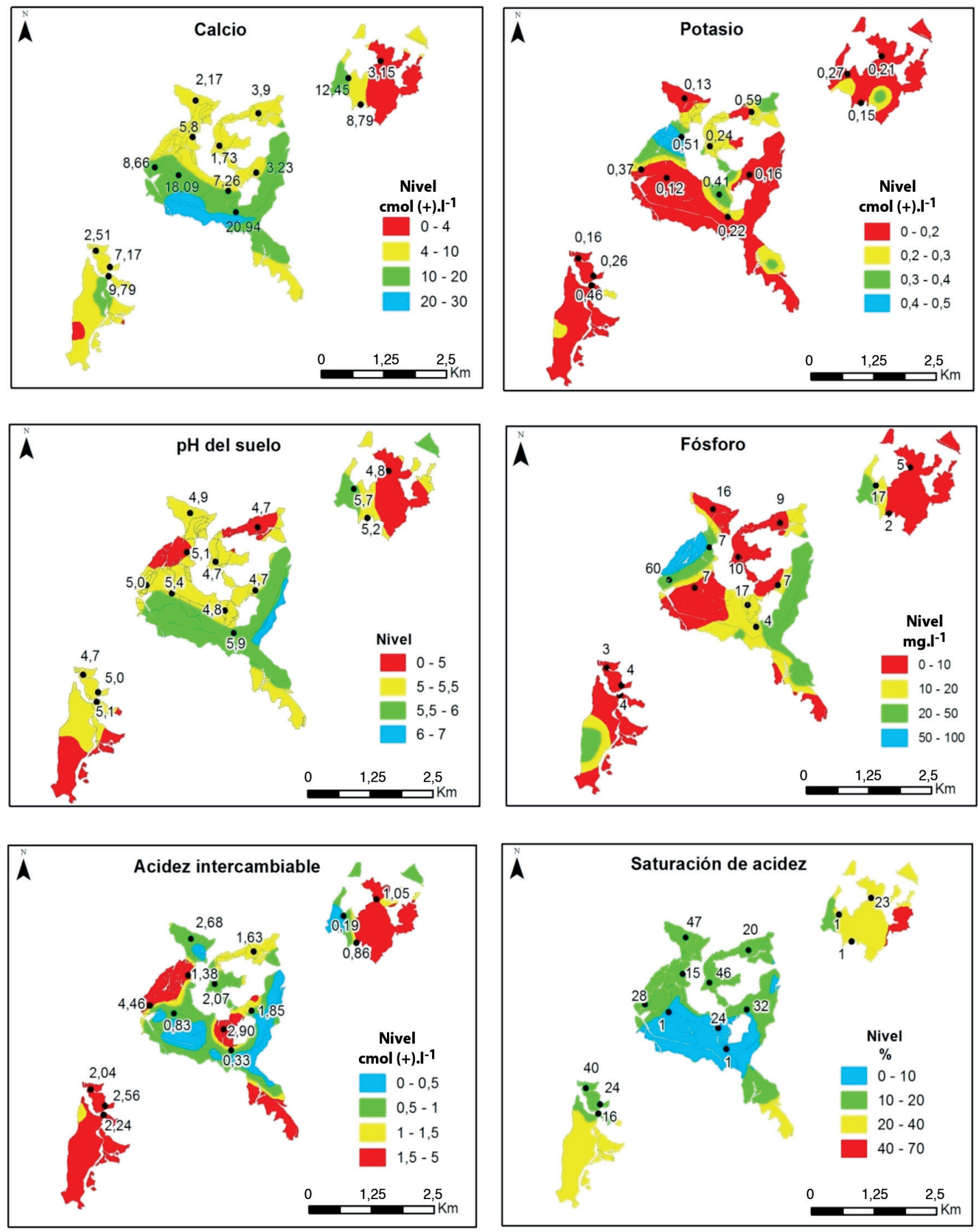

Fig. 2. Mapas interpolados y valores de verificación para Ca, K, pH, P, acidez intercambiable y \% de saturación de acidez del suelo para el área de estudio, Atirro, Costa Rica. 
la interpolación (Mueller et ál. 2001, Villatoro et ál. 2008). La estimación de la eficiencia de predicción (E) se hizo por medio de la siguiente formula:

$E=\left(\frac{\sum_{i=1}^{n_{v}} \tilde{\mathrm{V}} i^{2}}{n_{v}}-\frac{\sum_{i=1}^{n_{v}} V^{2}}{n_{v}} / \frac{\sum_{i=1}^{n_{v}} \tilde{\mathrm{V}}^{2}}{n_{v}}\right) * 100$

donde $\tilde{V}$ es la diferencia entre el valor obtenido en un punto y el promedio de todos los valores del set de datos, $V$ es la diferencia entre el valor de predicción y el observado en un punto $i$ ( $\mathrm{I}=1, \ldots$, $\mathrm{n}_{\mathrm{v}}$ ). y $n_{v}$ el número de puntos de validación.

Como criterios adicionales de validación se estimaron los porcentajes de acierto al tomar como referencia el traslape de los valores del muestreo de verificación dentro del rango de variación de laboratorio reportado por Corrales et ál. (2005); igualmente el otro criterio fue el porcentaje de acierto al ser la referencia el traslape de los valores del muestreo de verificación dentro del rango según las clases agronómicas establecidas en la simbología cartográfica de los mapas de interpolación con base en valores de referencia de la literatura (Bertsch 1995). En ambos casos se utilizaron los valores obtenidos del segundo grupo de datos (15 muestras).

\section{RESULTADOS Y DISCUSIÓN}

Los resultados encontrados muestran que el área de estudio presentó condiciones de variabilidad en tipo de suelo, topografía y manejo agrícola, aspectos que permitieron definir zonas con características diferentes entre sí, ideales para la realización de una investigación de este tipo particularmente con variables regionalizadas (Henríquez et ál. 2005).

Los parámetros geoestadísticos "Nugget", "Sill" y "Range" (en sus nombres en inglés, o bien "Efecto pepita", "Meseta" y "Alcance" en su traducción en español) de los semivariogramas generados y con los cuales se realizó la interpolación para cada una de las variables de estudio, se muestran en el Cuadro 1. En particular la variable P, presentó el valor de "Nugget" más grande según los valores del semivariograma. El mismo cuadro muestra que Ca y pH tuvieron el mayor "Range", lo que significa que son características de menor variabilidad y que mantienen su autocorrelación espacial en mayores distancias, comparativamente con las otras variables del estudio; ejemplo de ello es lo que ocurre con la acidez y \% de saturación con las cuales la autocorrelación se pierde en valores cercanos a los $400 \mathrm{~m}$ (Cuadro 1).

Como se puede observar los valores más bajos que se obtuvieron en el coeficiente de regresión $\left(\mathrm{R}^{2}\right)$ correspondieron a las variables $\mathrm{K}$ y $\%$ de saturación de acidez con un valor de 0,55 ; mientras que las variables que presentaron un mejor ajuste fueron el $\mathrm{Ca}$ y $\mathrm{pH}$, con valores de 0,82 y 0,79 respectivamente, lo cual concuerda con lo reportado por Villatoro et ál. (2008), ya que fueron las que mejor correlación presentaron al realizar la interpolación por medio del método Kriging ordinario. Los modelos que ajustaron mejor fueron el esférico y el Gausiano.

En los mapas generados a partir del análisis de interpolación Kriging de las variables de fertilidad utilizadas, presentados en la Figura 2 y basados en los parámetros geoestadísticos del Cuadro 1, se muestran también las clases establecidas con criterio agronómico para cada variable, así como la ubicación y los resultados puntuales de las variables de fertilidad de las 15 muestras que se utilizaron para la etapa de validación de campo.

Al utilizar el método de validación cruzada, se encontraron valores que difirieron a los obtenidos por el método de validación de campo, particularmente en relación con el \% de saturación de acidez, el cual fue mayor comparativamente en la primera. En este caso los coeficientes de correlación fueron para 0,83 para $\mathrm{Ca}, 0,66$ para $\mathrm{pH}, 0,63$ para \% de saturación de acidez, 0,56 para $\mathrm{K}, 0,56$ para acidez, 0,52 para $\mathrm{P}$ (Cuadro 2). 
Cuadro 2. Valores de Eficiencia de Predicción (E) y del coeficiente de correlación (r) obtenidos por medio de los métodos de "validación de campo" y "validación cruzada" para las variables de suelo analizadas.

\begin{tabular}{c|cccc}
\hline \multirow{2}{*}{ Variable } & \multicolumn{2}{c}{ Validación campo } & \multicolumn{2}{c}{ Validación cruzada } \\
\hline $\mathrm{pH}$ & $\mathrm{E}$ & $\mathrm{r}$ & $\mathrm{E}$ & $\mathrm{r}$ \\
$\mathrm{Ca}$ & 72 & $0,87 *$ & 70 & $0,83 *$ \\
$\mathrm{~K}$ & 42 & $0,78 *$ & 43 & $0,66 *$ \\
$\mathrm{P}$ & 10 & $0,44 \mathrm{~ns}$ & 31 & $0,56 *$ \\
Acidez & -3 & $0,65 *$ & 30 & $0,56 *$ \\
$\% \mathrm{SA}$ & 32 & $0,59 *$ & 27 & $0,52 *$ \\
\hline
\end{tabular}

*diferencia significativa a p menor a 0,05 aplicando la prueba de correlación simple Pearson.

En las Figuras 3 y 4 se presenta en forma gráfica la distribución de datos al comparar los datos estimados con los obtenidos con la aplicación de cada técnica. Se debe recordar que en el análisis de validación cruzada se trabaja con 138 datos dentro de una matriz de análisis interno de esos mismos 138 resultados; la validación de campo es una comparación entre los valores encontrados de un set de datos independiente contra los mapas generados a partir del set original de datos. El método de validación de campo presentó en general valores más altos de correlación (r) aunque en el caso de la acidez intercambiable y \% SA fue particularmente bajo, en tanto que el método de validación cruzada fue más constante en este índice. La técnica de validación cruzada en términos generales, mostró valores más favorables en la eficiencia de la estimación (E) que la técnica de validación de campo (Cuadro 2). En el caso del primer método de validación, los mejores coeficientes se obtuvieron con las variables $\mathrm{Ca}, \mathrm{pH}, \mathrm{K}$ y $\mathrm{P}(0,87,0,78,0,65$, 0,59 respectivamente), mientras que la acidez y $\%$ de saturación de acidez obtuvieron los coeficientes más bajos $(0,44$ y 0,09 respectivamente). 

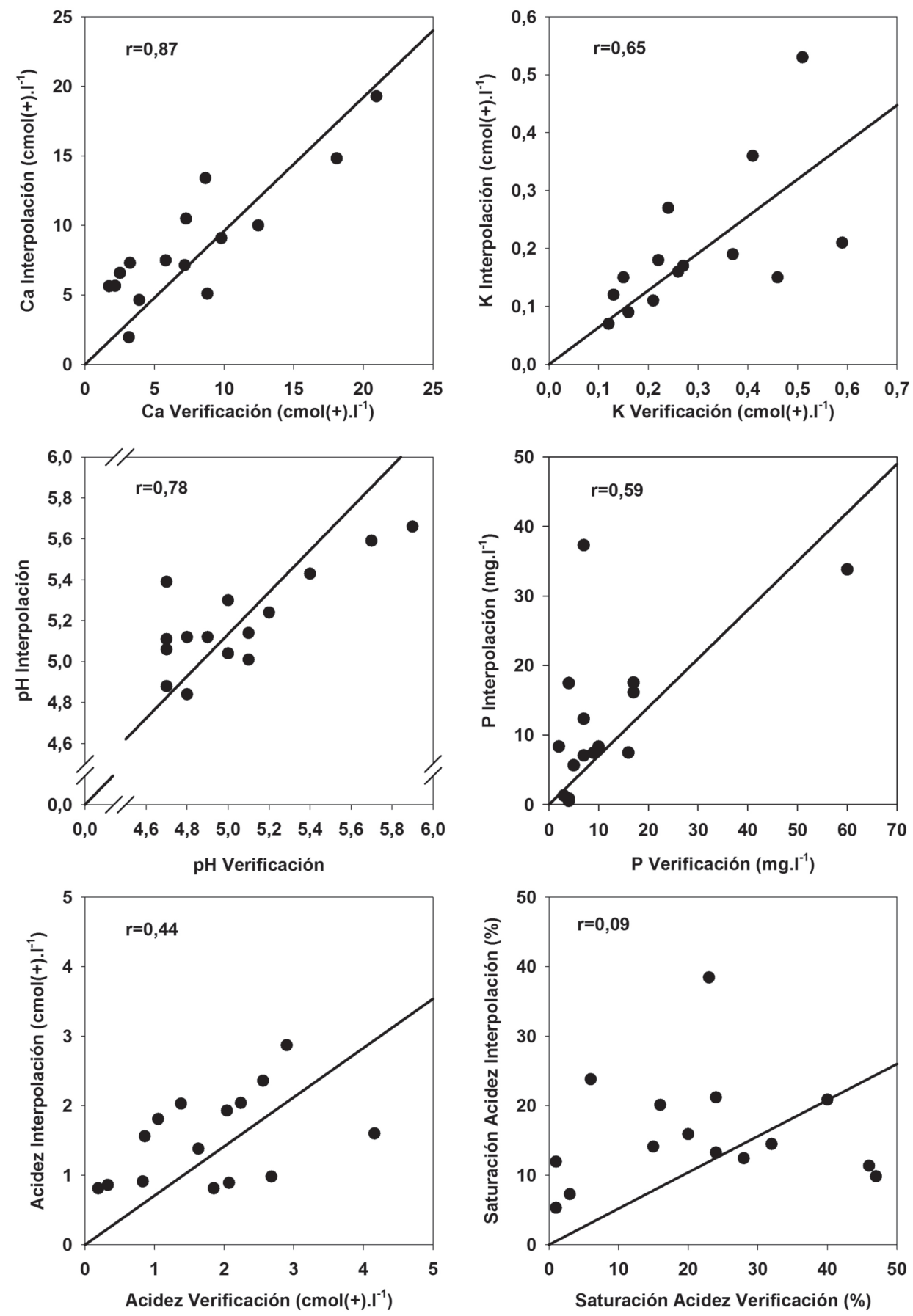

Fig. 3. Correlación entre los valores medidos y los interpolados para las diferentes variables de fertilidad de suelo con el método de validación de campo. Atirro, Costa Rica. 

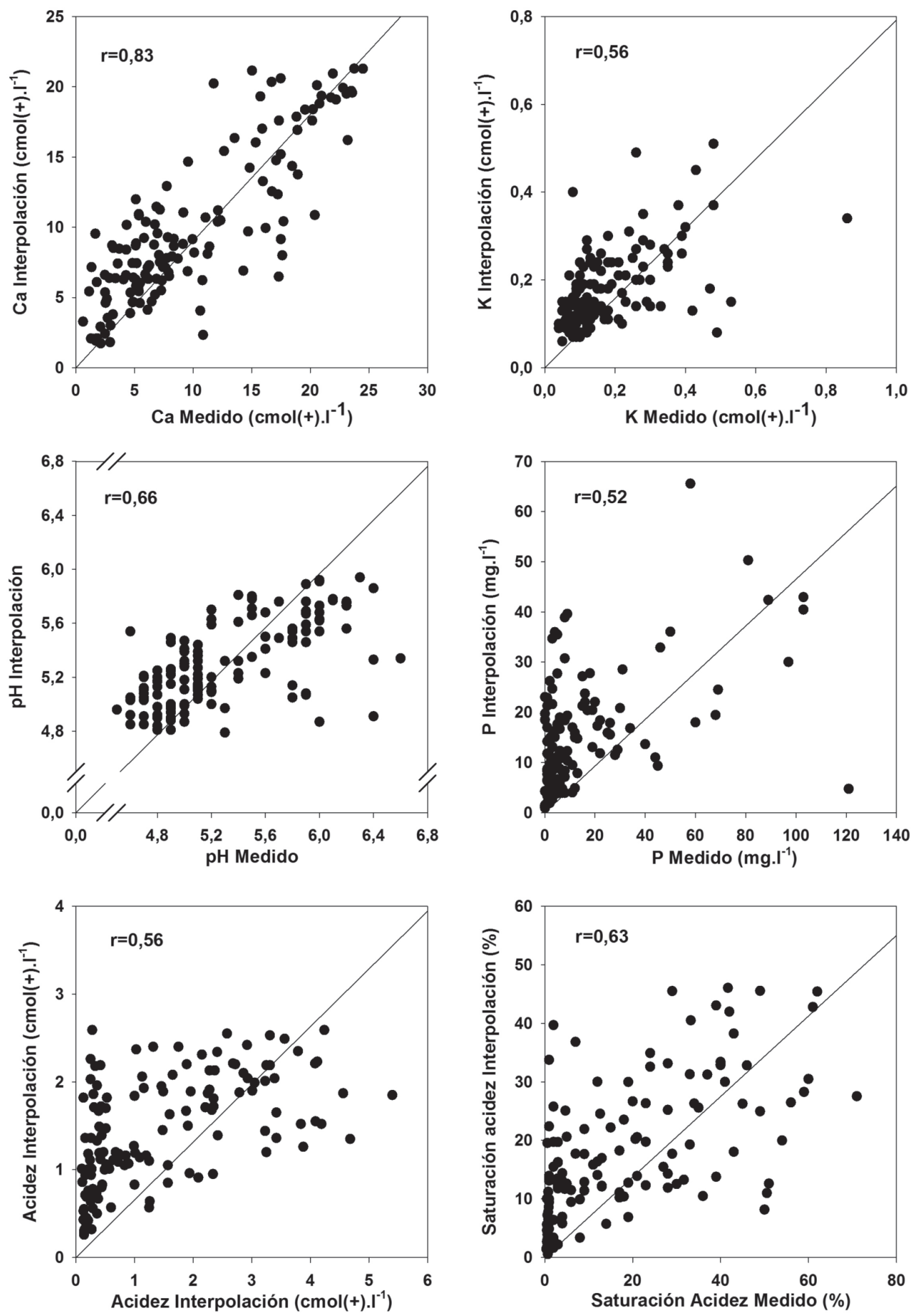

Fig. 4. Correlación entre los valores medidos y los interpolados para las diferentes variables de fertilidad de suelo con el método de validación cruzada. Atirro, Costa Rica. 
El valor promedio y los ámbitos de variación del análisis químico de suelo para cada variable del muestreo de verificación, así como los valores de predicción provenientes de los mapas interpolados, se presentan en el Cuadro 3. Como se mencionó anteriormente, para definir estos ámbitos de variación (entre el promedio y los valores máximo y mínimo) se utilizaron los porcentajes de variación de laboratorio para análisis químico de suelos reportados por Corrales et ál. (2005), los cuales en el caso de las variables utilizadas en este estudio fueron $\pm 15 \%$ para Ca y K, $\pm 5 \%$ para $\mathrm{pH}, \pm 30 \%$ para $\mathrm{P}, \pm 25 \%$ para acidez y $\%$ SA. Esta variación se aplicó tanto a los valores del análisis de laboratorio del muestreo de verificación como a los valores de predicción de la interpolación de Kriging con el fin de equiparar un criterio de comparación entre ambas variables.

Cuadro 3. Valores promedio, rango de variación, desviación estándar y coeficiente de variación de los parámetros estadísticos de los valores reales y de interpolación obtenidos en los puntos de verificación.

\begin{tabular}{|c|c|c|c|c|c|c|c|c|c|c|}
\hline \multirow{2}{*}{ Variable } & \multicolumn{5}{|c|}{ Valor real } & \multicolumn{5}{|c|}{ Valor de interpolación } \\
\hline & $\mathrm{X} \min$ & $\mathrm{X}$ & $\mathrm{X} \max$ & $\mathrm{DE}$ & $\mathrm{CV}$ & $\mathrm{X} \min$ & $\mathrm{X}$ & $\mathrm{X} \max$ & $\mathrm{DE}$ & $\mathrm{CV}$ \\
\hline $\mathrm{Ca}$ & 6,55 & 7,71 & 8,87 & 5,76 & $75 \%$ & 7,32 & 8,61 & 9,90 & 4,43 & $51 \%$ \\
\hline $\mathrm{pH}$ & 4,79 & 5,05 & 5,30 & 0,37 & $7 \%$ & 4,90 & 5,16 & 5,42 & 0,33 & $6 \%$ \\
\hline Acidez & 1,34 & 1,78 & 2,23 & 1,06 & $60 \%$ & 1,10 & 1,47 & 1,84 & 0,88 & $60 \%$ \\
\hline $\mathrm{K}$ & 0,24 & 0,28 & 0,33 & 0,15 & $52 \%$ & 0,15 & 0,18 & 0,21 & 0,14 & $75 \%$ \\
\hline $\mathrm{P}$ & 8,03 & 11,47 & 14,91 & 14,33 & $125 \%$ & 8,13 & 11,62 & 15,10 & 12,02 & $103 \%$ \\
\hline$\% \mathrm{SA}$ & 16,30 & 21,73 & 27,17 & 16,71 & $70 \%$ & 11,56 & 15,41 & 19,26 & 12,22 & $79 \%$ \\
\hline
\end{tabular}

Como también se puede observar en los mapas de la Figura 2 así como en el Cuadro 4, hubo un mayor porcentaje de acierto con respecto a los ámbitos establecidos por clase agronómica que por la incertidumbre misma del análisis. El porcentaje de acierto entre los puntos de verificación y las clases agronómicas definidas en los mapas osciló entre 47 y $93 \%$, al ser el pH y el $\mathrm{P}$ las variables que con mayor porcentaje de acierto presentaron con la utilización de esta herramienta o criterio (Cuadro 4).

Con base en lo anterior, se logró también determinar otro criterio de validación el cual fue el porcentaje de acierto obtenido a partir del traslape con los rangos generados al considerar la diferencia entre los promedios de las 15 muestras de verificación y los valores de predicción. Estos porcentajes de acierto variaron entre 27 y $93 \%$ al ser el $\mathrm{K}$ y el \% SA, las variables que presentaron el menor de estos valores y el $\mathrm{pH}$ la que mejor resultado obtuvo según este criterio de validación (Cuadro 4).

Cuadro 4. Resultados de la prueba de validación por los métodos de porcentaje de acierto por rango estadístico (variación de análisis) y por clase agronómica de las variables de fertilidad de suelo en Atirro, Costa Rica.

\begin{tabular}{c|cc}
\hline \multirow{2}{*}{ Variable } & \multicolumn{2}{c}{$\%$ de acierto } \\
\cline { 2 - 3 } & Por rango & Por clase \\
\hline $\mathrm{Ca}$ & 47 & 60 \\
$\mathrm{pH}$ & 93 & 93 \\
Acidez & 60 & 53 \\
$\mathrm{~K}$ & 27 & 60 \\
$\mathrm{P}$ & 53 & 73 \\
\% SA & 27 & 47 \\
\hline
\end{tabular}




\section{CONCLUSIÓN}

Los datos obtenidos en este estudio a partir de los 2 tipos de validación utilizados, así como los 2 métodos comparativos, sugieren que los mapas generados a partir de un proceso de interpolación a una escala regional, pueden ser una herramienta útil como aproximación y referencia para predecir con un buen grado de acierto las propiedades de fertilidad de suelos a ese nivel, según referencias señaladas en el Cuadro 4 sobre resultados de la prueba de validación por los métodos de porcentaje de acierto por rango estadístico (variación de análisis) y por clase agronómica de las variables de fertilidad de suelo en Atirro, Costa Rica. Pese a ello, se sugiere que la aplicación de un proceso de validación posterior es indispensable para confirmar el grado de precisión de los mapas generados a partir de esta herramienta. En este caso particular, el proceso de validación de campo puede presentar ventajas comparativas, al ser un proceso totalmente independiente a la generación original de los mapas de interés y puede ser tomado como un proceso de corroboración de mucha confiabilidad.

\section{AGRADECIMIENTOS}

Los investigadores agradecen al Instituto de Fomento Cooperativo (INFOCOOP) por facilitar los fondos requeridos para llevar a cabo esta investigación. Asimismo se agradece a las cooperativas participantes por su apoyo logístico a nivel de campo para la toma de muestras así como por facilitar el acceso.

\section{LITERATURA CITADA}

BERTSCH F. 1995. Fertilidad de suelos y su manejo. San José, Costa Rica. Asociación Costarricense de la Ciencia del Suelo (ACCS). 157 p.

BERTSCH F., HENRÍQUEZ C., RAMÍREZ F., SANCHO F. 2002. Site-Specific nutrient management in the highlands of Cartago province. Better Crops International 16(1):16-19.

CLAY D.E., KITCHEN N., CARLSON C.G., KLEINJAN J., CHANG H. 2007. Using historical management to reduce soil sampling errors, pp. 49-64. In: D. Clay and F. Pierce (ed.). GIS Application in Agriculture. CRC Press.

CORRALES M., BERTSCH F., BEJARANO J. 2005. Los laboratorios de análisis de suelos y foliares en Costa Rica: Informe del Comité de Laboratorios de análisis de suelos, plantas y aguas. Agronomía Costarricense 29(3):125-135.

DIAZ R., HUNTER A. 1978. Metodologías de muestreo de suelos, análisis químico de suelos y tejido vegetal y de investigación en invernadero. Turrialba, Costa Rica. CATIE. 62 p.

GALLARDO A. 2006. Geostadística. Ecosistemas 15(3):48-58.

HENRÍQUEZ C., KILLORN R., BERTSCH F., SANCHO F. 2005. La geostadística en el estudio de la variación espacial de la fertilidad del suelo mediante el uso del interpolador kriging. Agronomía Costarricense 29(2):73-81.

MUELLER T.G. 2007. Map quality assessment for sitespecific fertility management, pp.103-120. In: D. Clay and F. Pierce (ed.). GIS Application in Agriculture. CRC Press.

PETERSEN G.W., BELL J.C., MCSWEENEY K., NIELSEN G.A., ROBERT P.C. 1995. Geographic information systems in agronomy. Advances in Agronomy 55:67-105.

ROBINSON T.P., METTERNICHT G. 2006. Testing the performance of spatial interpolation techniques for mapping soil properties. Computers and Electronics in Agriculture 50:97-108.

TOMCZAK M. 1998. Spatial interpolation and it uncertainty using automated anisotropic inverse distance weighting (IDW) cross validation-Jackknife approach. Journal of Geographic Information and Decision Analysis 2(2):18-30.

TRANGMAR B.B., POST R.S., UEHARA G. 1985 Application of geostatistics to spatial studies of soil properties. Advances in Agronomy. 38:45-94.

USDA. 2010. Keys to Soil Taxonomy, 11th ed. USDANatural Resources Conservation Service, Washington, DC. 365 p.

VILLATORO M., HENRÍQUEZ C., SANCHO F. 2008 Comparación de los interpoladores IDW y Kriging en la variación espacial de $\mathrm{pH}, \mathrm{Ca}, \mathrm{CICE}$ y $\mathrm{P}$ del suelo. Agronomía Costarricense 32(1):95-105.



Todos los derechos reservados. Universidad de Costa Rica. Este artículo se encuentra licenciado con Creative Commons Reconocimiento-NoComercial-SinObraDerivada 3.0 Costa Rica. Para mayor información escribir a rac.cia@ucr.ac.cr 